Article

\title{
Conductometric Sensor for PAH Detection with Molecularly Imprinted Polymer as Recognition Layer ${ }^{+}$
}

\author{
Usman Latif ${ }^{1,2}$ (D), Liu Ping ${ }^{1}$ and Franz L. Dickert ${ }^{1, *(1)}$ \\ 1 Department of Analytical Chemistry, University of Vienna, Währinger Strasse 38, Vienna A-1090, Austria; \\ liupingsjj@sjtu.edu.cn \\ 2 Interdisciplinary Research Centre in Biomedical Materials (IRCBM), COMSATS Institute of Information \\ Technology (CIIT), Lahore 54000, Pakistan; usmanlatif@ciitlahore.edu.pk \\ * Correspondence: franz.dickert@univie.ac.at; Tel.: +43-1-4277-52301 \\ + Dedicated to Prof. Dr. Wolfgang Kautek on the occasion of his 65th birthday.
}

Received: 27 December 2017; Accepted: 28 February 2018; Published: 3 March 2018

\begin{abstract}
A conductometric sensor based on screen-printed interdigital gold electrodes on glass substrate coated with molecularly imprinted polyurethane layers was fabricated to detect polycyclic aromatic hydrocarbons (PAHs) in water. The results prove that screen-printed interdigital electrodes are very suitable transducers to fabricate low-cost sensor systems for measuring change in resistance of PAH-imprinted layers while exposing to different PAHs. The sensor showed good selectivity to its templated molecules and high sensitivity with a detection limit of $1.3 \mathrm{nmol} / \mathrm{L} \mathrm{e.g.,} \mathrm{for} \mathrm{anthracene} \mathrm{in}$ water which is lower than WHO's permissible limit.
\end{abstract}

Keywords: conductometric sensor; screen-printing; interdigital gold electrodes; molecularly imprinted polymers; polycyclic aromatic hydrocarbons

\section{Introduction}

There is a huge demand for environmental monitoring technologies due to increasing environmental pollutants and their adverse health effects. PAHs are ubiquitous environmental contaminants mainly from incomplete combustion of various materials, especially from petroleum. PAHs are a group of persistent organic pollutants and bioaccumulatives [1]. They are moderate-tohighly toxic due to their mutagenic, teratogenic, and carcinogenic properties [2,3]. PAHs contains at least two aromatic rings. Anthracene, a PAH consisting of three fused benzene rings, is deleterious to human health and can cause lung, liver, lymph, and skin disorders [4]. The prime importance has been given, in the latest European Water Framework Directives (Directives 2000/60/EC, 2006/118/EC, and 2006/11/EC), to develop those analytical tools which are useful for sensitive and on-site detection of pollutants in environmental waters [5].

Existing techniques for detecting PAHs rely on bulky instruments which require sample preparation, its isolation and large volume of organic solvents that make these methods very time consuming and costly. There are some reports about coupling selective extractions with instrumental analysis to detect PAHs [6-13]. Trace analytical methods for complex matrices rely on efficient sample enrichment and selective assays. These methods are very sensitive but ill-suited for on-site monitoring of pollutants due to their limitations. Chemical sensors have emerged as a powerful tool for special analytes using artificial recognition sites [14]. In the search for new recognition techniques, molecular imprinted polymers (MIP) have opened a way to a novel type of selective sorbent with attractive properties. On-line monitoring of small organic pollutants in waters is feasible with MIPs [15-21]. Membrane-based conductometric sensors for various target compounds have also 
been reported [22-24]. Imprinted layers of modified non-porous or porous particles, films, or other materials can be used to detect various analytes. Non-covalent imprinting can be easily performed for small molecules with no functionality such as PAHs. The imprinting effect depends preferably on van der Waals forces between template and self-organizing polymers.

In this study, MIP based conductometric sensor has been developed for anthracene with a detection limit of $1.3 \mathrm{nmol} / \mathrm{L}$ which is lower than WHO's permissible limit of PAHs ( $3.93 \mathrm{nmol} / \mathrm{L})$ [25].

\section{Materials and Methods}

Pre-cleaned, plain, cut edges microscope glass slides $(76 \mathrm{~mm} \times 26 \mathrm{~mm} \times 1 \mathrm{~mm})$ were purchased from Marienfeld. Gold paste (GGP 2093, 12\%) for electrode printing was received from Heraeus, Germany. The isolating paste (Urethan 71) was purchased from CRC Industries. PAHs were purchased from Fluka and Sigma. Bisphenol A (BPA), 4,4'-methylenediphenyldiisocyanate (MDI), tetrahydrofuran (THF) and dimethylsulfoxide (DMSO), and phloroglucinol (PG) were purchased from Merck and Fluka. 3-aminopropyltriethoxysilane was obtained from ABCR. Analytical grade chemicals were obtained and used as received.

\subsection{Fabrication and Surface Modification of Electrodes}

Interdigital electrodes (IDE) were screen-printed on a glass substrate (microscope slide) to fabricate an interdigital transducer (IDT). The equivalent circuit diagram and working principle of IDT was already explained in our previous paper [24]. Screen-printed interdigitated gold electrode-based sensor systems have been used for variety of analytes [26-28]. The desired structure of interdigital electrodes was fabricated on the substrate via screen printing with commercial gold paste. The type of the screen was 120-34W PW of Sefar Inc. (Thal, Switzerland). The electrode structure was designed by photo lithography. All organic components present in gold paste were removed by heating at $500{ }^{\circ} \mathrm{C}$ for $4 \mathrm{~h}$. The advantage of this procedure is based on the robustness of the gold layers. The pattern and dimensions of the IDEs structure on transducer surface is shown in Figure 1.

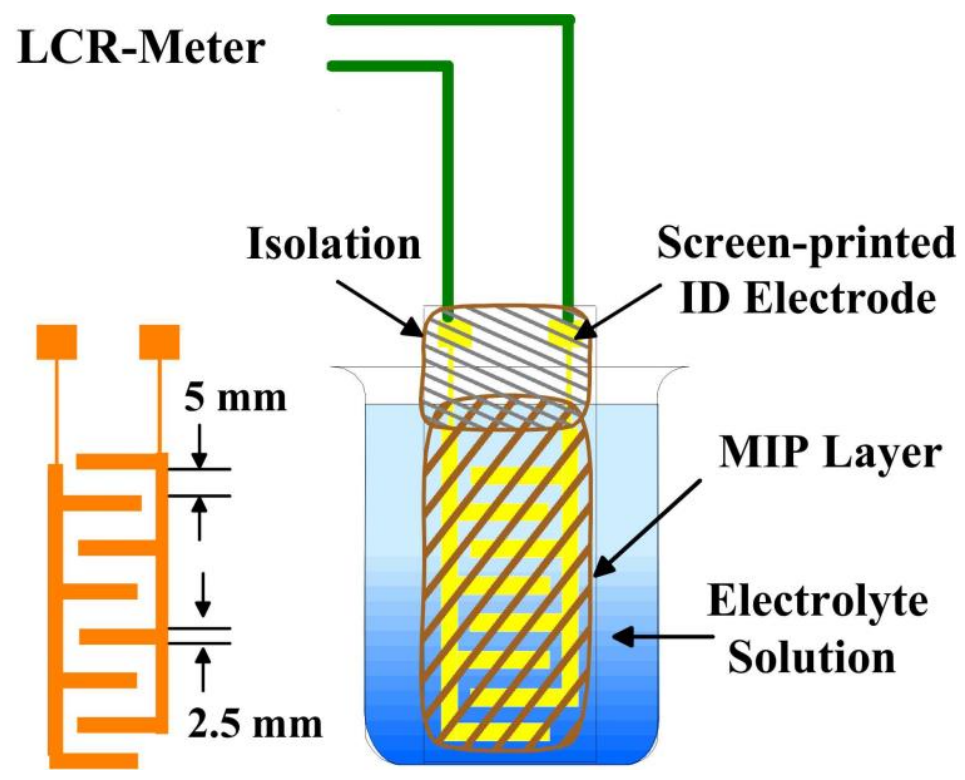

Figure 1. IDT was fabricated by screen printing IDE of gold on glass substrate. IDT was coated with sensitive layer and LCR meter was used to measure resistance of sensitive layer by placing it in glass cell filled with electrolyte solution.

The prepared transducer was cooled down to room temperature, then washed in an ultrasonic water bath for $5 \mathrm{~min}$ and dried. The IDT was placed in a boiling mixture of chloroform and ethanol 
$(1: 1 \mathrm{v} / \mathrm{v})$ for at least $10 \mathrm{~min}$ for washing. Afterwards, IDT was treated with hot lithium hydroxide solution $(0.2 \mathrm{mM})$ for $10 \mathrm{~min}$, rinsed with distilled water and dried. Then, IDT was dipped in a solution of 3-aminopropyl-triethoxysilane in ethanol for 1-2 $\mathrm{min}$ followed by rinsing with ethanol. The silanized structure was cured for $10 \mathrm{~min}$ at $110^{\circ} \mathrm{C}$.

Molecular imprinted polyurethane layers were synthesized by following the procedure from our previous paper [14]. BPA, PG, and MDI containing 30\% of the respective triisocyanate (mixture of isomers) and $5-10 \%$ of $\mathrm{PAH}$ as imprint were dissolved in THF to prepare $\mathrm{PAH}$-imprinted polyurethane layer. The pre-polymerized solution $\left(70^{\circ} \mathrm{C}\right.$ for $\left.1 \mathrm{~h}\right)$ from above mentioned mixture was spin-coated on IDT surface. A mixture of monomers, cross-linker, and template was heated at $70{ }^{\circ} \mathrm{C}$ until its gelation point which is called as pre-polymer. This pre-polymer was applied to transducer surface to form a rigid layer after complete polymerization. The height of the coating can be controlled by the rotation speed of the spin coating apparatus, which was home-made. Additionally, the viscosity of the pre-polymer can be varied by dilution, which has the same effect as higher rotation speed. The highly cross-linked prepolymer formed a rigid clear transparent homogenous layer on IDT surface after complete condensation. The surface condition and heights of layers were analyzed by using atomic force microscope (VEECO Nanoscope IVa) in contact mode. The electrodes were connected with Hewlett-Packard 4284 A precision LCR meter, the uncoated area was finally isolated with urethane 71. The reference transducer was prepared with non-imprinted polyurethane in exactly same way (these are called 'non-imp. electrodes').

\subsection{Removal of the Template Molecule}

We have used different ways to remove imprinted molecule from MIP layers such as washing with toluene, rinsing with water, and evaporating using hot air. The imprinted layer, containing high boiling point PAHs or low evaporation rates, was washed by toluene and water. It should be very careful to control the washing time of toluene, otherwise toluene can damage the cavity structure in MIPs layer. Whereas, rest of PAHs (templates) were easily removed by evaporating at elevated temperatures. The template molecules were evaporated from MIPs by using hot air $\left(28^{\circ} \mathrm{C}\right)$. It should be given enough evaporating time to generate diffusion pathways while removing template molecules, during polymerization, otherwise diffusion pathways will close (if template evaporate immediately) when polymer is still rearranging itself. The imprinted polymer layer containing $10 \%$ naphthalene as template, with different evaporating times was examined by luminescence spectrometer (Perkin-Elmer LS-50B). The results can be seen in Table 1. This method is suitable for removing both naphthalene and anthracene from MIP layer. In general, cavities in MIPs were almost fully recovered after $4 \mathrm{~h}$ of evaporation. The imprinted layers were stored in an evacuated desiccator to accelerate evaporation

Table 1. Comparison of fluorescence intensity (Perkin-Elmer LS-50B-spectrometer) of $10 \%$ naphthalene imprinted polymer layer at different evaporation times.

\begin{tabular}{ccccccc}
\hline Polymer & Non-Imprinted & \multicolumn{5}{c}{ 10\% Imprinted Layer } \\
\hline Time [hour] & 0 & 0 & 0.5 & 1.5 & 2.5 & 4 \\
$\mathrm{I} / \mathrm{I}_{0}[\%]$ & $2.5 \%$ & $100 \%$ & $50 \%$ & $27 \%$ & $21 \%$ & $5 \%$ \\
\hline
\end{tabular}

I: fluorescence intensity of $10 \%$ naphthalene imprinted polymer layer after evaporation or non-imprinted layer; $\mathrm{I}_{0}$ : fluorescence of freshly prepared $10 \%$ naphthalene imprinted polymer layer. $2.5 \%$ : percentage of fluorescence intensity of non-imprinted polymer layer to $10 \% \mathrm{PAH}$ imprinted polymer layer (having same layer thickness).

\subsection{Measurements}

The resistance of the sensitive layer (after template removal) coated on IDT was measured by placing it in a glass cell having a supporting electrolyte as shown in Figure 1. The stock solution of analytes was added into the glass cell having $50 \mathrm{~mL}$ of supporting electrolyte to obtain final medium. Hewlett-Packard 4284A precision LCR meter $(20 \mathrm{~Hz}-1 \mathrm{MHz})$ was operated in an AC mode to measure 
resistance of sensitive layers. The surface and the thickness of the MIP layers were measured with a VEECO Nanoscope IVa atomic force microscope.

\section{Results and Discussion}

\subsection{Thickness of MIP Layers}

The polyurethane layers were imprinted with anthracene and applied to transducer surface in a height ranging from $250 \mathrm{~nm}$ to approximately $1 \mu \mathrm{m}$. The transducer surface was covered with MIP layers by spin-coating prepolymer solution. Required thickness was achieved by adjusting speed according to solution viscosity. All spin-coated layers were homogeneous, transparent, and highly stable. The absolute resistance due to analyte enrichment increases with layer thickness. A layer in a height of $250 \mu \mathrm{m}$ will have a resistance of approximately $20 \mathrm{k} \Omega$, whereas coatings in a thickness of some micro meter show resistances of several $\mathrm{M} \Omega$. The stated enrichment process with blocked diffusion channels is a plausible reason for this sensor effects [29]. In summary, the thickness of sensitive layer is an essential factor in conductometric measurements. Here, $100 \mathrm{~nm}$ up to $1 \mu \mathrm{m}$ layers are considered as an optimum thickness of PAH imprinted polyurethane layer in this conductometric sensor measurements, since these coatings were rigid without cracks.

The surface morphology of sensor coatings was analyzed by using AFM in contact mode. Figure 2 shows a $10 \%$ anthracene imprinted homogeneous polyurethane layer. This depicts section analysis of scratched surface layer to measure its thickness, which is around $1 \mu \mathrm{m}$.

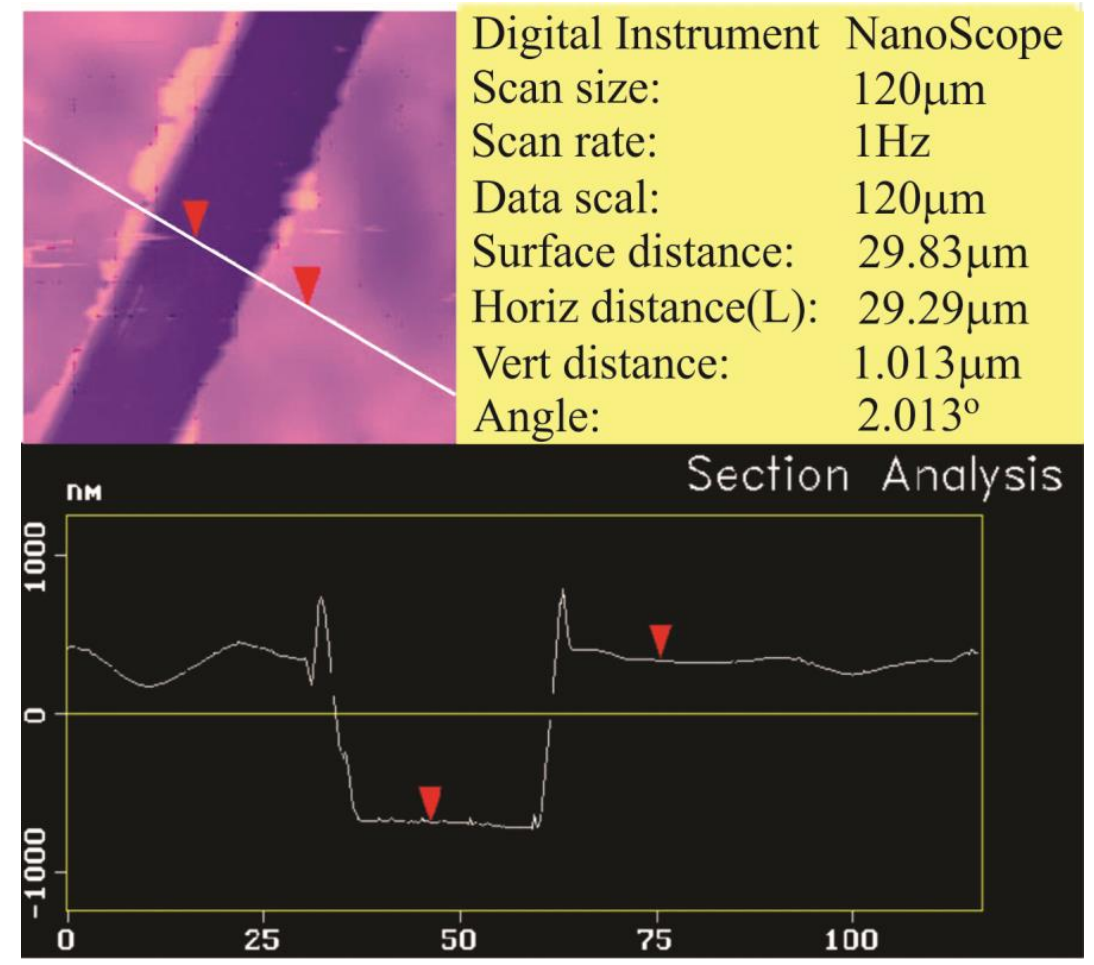

Figure 2. The thickness of $10 \%$ anthracene imprinted polymer layer measured by contact mode of AFM.

\subsection{Optimization of Measurement Conditions}

The resistance of IDT covered with 10\% anthracene imprinted polyurethane layer in aqueous $\mathrm{NaCl}$ solution (Figure 3) was measured with LCR meter at a frequency of $1 \mathrm{kHz}$. It was found that the resistance of MIP layer increased due to analyte incorporation in imprinted cavities of sensor coating as function of time. The increase in resistance is based on incorporation of analytes into the cavities of imprinted polymers. In this way, diffusion channels are closed and cavities are 
properly filled, which leads to an increase in resistance. The resistance of anthracene imprinted layer increases with time which means templated analyte (anthracene) is continuously diffused into a large number of cavities. In this experiment, $10 \%$ anthracene was used as template to synthesize imprinted polyurethane layer. The sensor is regenerated within approximately one hour. It can be seen from Figure 3 that signal intensity compared to error bar is approximately 13:1. The IUPAC designates the ratio $3: 1$ as significant response. Thus it can be concluded that a limiting concentration for the detection of anthracene is $5.6 \mathrm{nM}(1 \mu \mathrm{g} / \mathrm{L}) 3 / 13=1.3 \mathrm{nM}$.

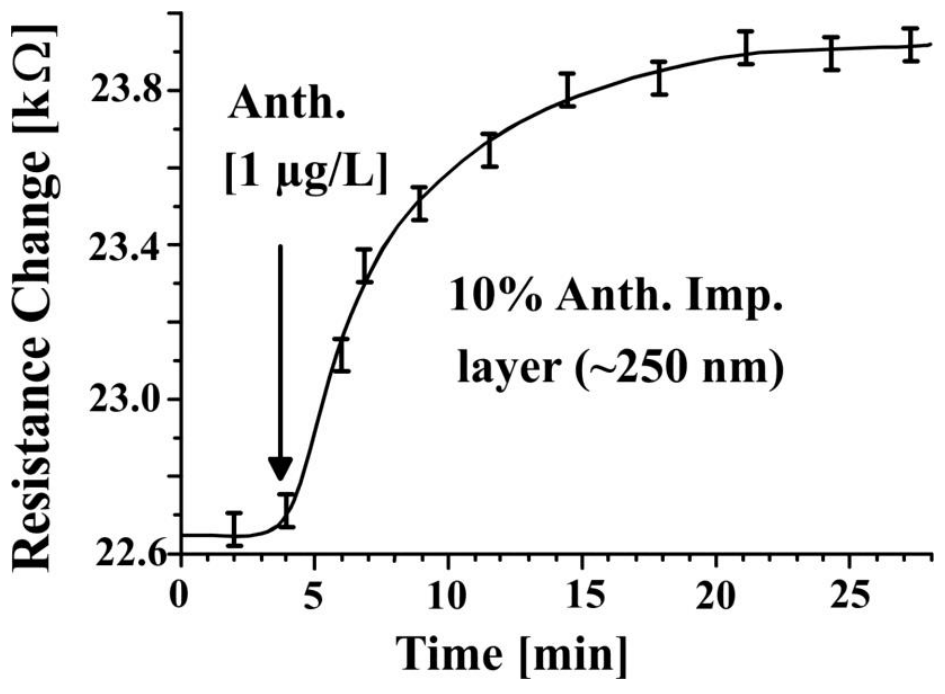

Figure 3. The resistance of $10 \%$ anthracene imprinted polyurethane layer (coated on IDT) measured with LCR meter at a frequency of $1 \mathrm{kHz}$ while exposing to its template analyte solution. The resistance of layer increases with time due to blocking of diffusion channels.

Selectivity of a $5 \%$ anthracene imprinted polyurethane layer $(100 \mathrm{~nm})$ based on the conductometric sensor is shown in Figure 4. The sensor layer yields the highest response to anthracene in comparison to other PAHs. Whereas minor sensor responses were observed for other PAHs, since they are not perfectly adapted or incorporated into receptor cavities of anthracene imprinted layer. Fewer changes occurred in resistance for the non-imprinted polyurethane layer $(100 \mathrm{~nm})$ due to absence of selective interaction sites.

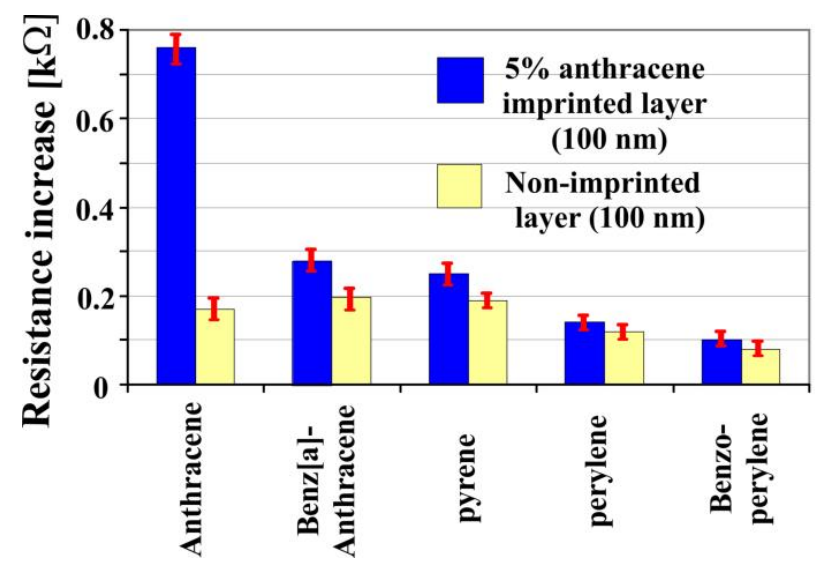

Figure 4. Anthracene imprinted layer was exposed to its templated analyte as well as other PAHs. The sensor showed higher sensor response to its templated analyte, but fewer responses were observed for other PAHs. 


\subsection{Influence of Physical Parameters to Sensor Response}

The IDTs were coated with layers ranging from $\sim 30 \mathrm{~nm}$ to $5 \mu \mathrm{m}$ with different PAH imprinted polyurethanes. The results indicate that the layers with a height of $100 \mathrm{~nm}$ to $1 \mu \mathrm{m}$ are best for resistance measurement in our experiment. The sensitivity of MIP layer to e.g., $2 \mu \mathrm{g} / \mathrm{L}$ analyte increased in parallel with the MIP layer thickness. Slightly enhanced response times, from 10-20 min, were observed while increasing the thickness of layer from $100 \mathrm{~nm}, 250 \mathrm{~nm}$ to $1 \mu \mathrm{m}$. The results were not reproducible while decreasing the thickness of layer below than $50 \mathrm{~nm}$. Layers exceeding the height of $5 \mu \mathrm{m}$ proved to be difficult in performing measurements due to high resistance.

The good wetting ability of polymer material is the basis of effects described. Thus, electrolytes can penetrate into the channels of the polymer materials. The ion mobility and its concentration will strongly influence the sensor basic response.

\subsection{Effect of Porogens}

The use of porogens or porogenic solvents during polymerization is a best way to produce effective molecular recognition capabilities in polymers, especially in non-covalent molecular imprinting. The chemical and physical properties of porogenic additives during polymerization will influence the rigidity and functionality of MIPs. The porogens will induce porosity in the resulting microstructure, which will increase better accessibility of analytes to binding sites. The porogens should not compete with template during imprinting and should not form complexes with polymers. THF is different from PAH molecules and allows a good solubility of the polyurethane compounds.

For this purpose, diphenylmethane (DPM) was added to the mixture of pre-polymer. Three $5 w / w \%$ anthracene imprinted polyurethane layers (MIP1, MIP2, MIP3) were prepared in a mixture of THF as solvent and DPM as porogen (the ratio of THF to DPM is 100:0, 98:2, and 90:10 respectively). These three anthracene imprinted layers show a characteristic response pattern to PAH molecules having different sizes and shapes as shown in Figure 5. Sensor coatings with MIP1 show appreciable responses to small PAHs such as naphthalene, anthracene, and phenanthrene. The molecular hollows will effectively bind the PAHs by which ion diffusion is hindered. In comparison to smaller PAHs, sensor response to bulky PAHs is lower. In accordance with these findings MIP2 layers show a pronounced sensitivity to bulky PAHs. The enlarged porosity will more strongly enrich bulky PAHs but not the small analogues. The further increase of porogens in MIP3 shows pronounced responses for bulky PAHs.

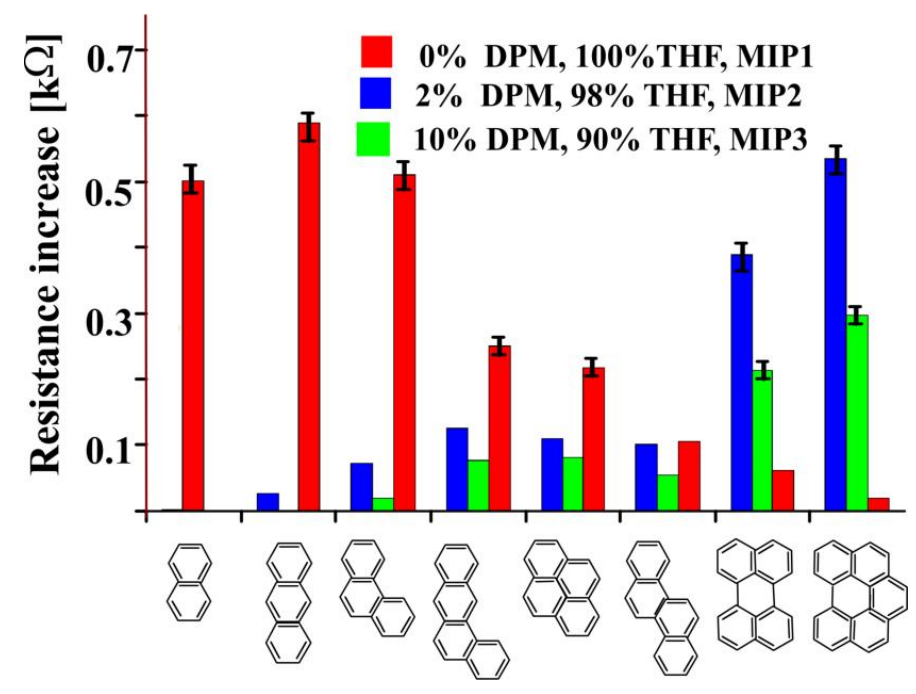

Figure 5. The effect of porogens on sensor responses of $5 \%$ anthracene imprinted polyurethane layer towards different PAHs. 
The resistances of sensitive layers were measured by placing them in a glass cell having supporting electrolyte. Thus, ion migration (from electrolyte) increases with porosity of polymer which results a decrease in resistance. The signal intensity, however, is smaller as for MIP2 since the increased porosity leads to a higher ion migration independent of the occupied or unoccupied cavities.

\subsection{Stability and Reproducibility}

The stability of MIP layer was tested over a period of two months by repeating measurements of its resistance to $2 \mu \mathrm{g} / \mathrm{L}$ anthracene in electrolyte solution. It was found that the sensor electrode which was stored at room temperature did not show any sensitivity loss over time.

The reproducibility of the sensor depends on the surface coating of electrode, the modification of screen-printed IDEs, and removal of template molecules. The reproducibility of MIP layer was achieved by controlling coating volume of fresh polymer on IDTs and speed of spin-coating.

\section{Conclusions}

PAH imprinted polyurethane coated conductometric sensors based on screen-printed interdigitated electrode were developed. A screen-printed IDE on glass substrate (microscope slide) was proven to be a suitable low cost and easily fabricated conductometric transducer. A sensor coated with $1 \mu \mathrm{m} \mathrm{10 \%}$ anthracene imprinted polymer layer shows the highest sensor response to the templated analyte. The detection limit to anthracene in $0.1 \mathrm{mM} \mathrm{NaCl}$ (as electrolyte) is $1.3 \mathrm{nM}$. The response time is about $20 \mathrm{~min}$. The sensor response depends on the nature and concentration of the electrolyte used during resistive measurements. Thus, the starting resistance must be known. Furthermore, the temperature has to be kept constant during resistive measurement. Comparing with other sensors, it exhibits the advantages of easy fabrication, good storage stability, good regeneration ability, low cost, and easy miniaturization.

Author Contributions: All authors have made significant contributions in this research article. Franz L. Dickert conceived the idea and Usman Latif designed the customized sensor setups for data acquisition. Liu Ping performed the experiments. Usman Latif and Franz L. Dickert contributed in paper writing and final version of manuscript was prepared after several rounds of critical revisions.

Conflicts of Interest: The authors declare no conflict of interest.

\section{References}

1. Woodland, W.; Motti, C.A.; Irving, P.; Van Herwerden, L.; Vamvounis, G. A colorimetric approach towards polycyclic aromatic hydrocarbon sensing. Aust. J. Chem. 2016, 69, 1292-1295. [CrossRef]

2. Wang, L.; Wan, X.M.; Gao, R.; Lu, D.F.; Qi, Z.M. Nanoporous gold films prepared by a combination of sputtering and dealloying for trace detection of benzo[a]pyrene based on surface plasmon resonance spectroscopy. Sensors 2017, 17, 1255. [CrossRef] [PubMed]

3. Du, J.; Jing, C. Preparation of thiol modified $\mathrm{Fe}_{3} \mathrm{O}_{4} @ \mathrm{Ag}$ magnetic SERS probe for PAHs detection and identification. J. Phys. Chem. C 2011, 115, 17829-17835. [CrossRef]

4. Makelane, H.; John, S.V.; Yonkeu, A.L.D.; Waryo, T.; Tovide, O.; Iwuoha, E. Phase selective alternating current voltammetric signalling protocol: Application in dendritic co-polymer sensor for anthracene. Electroanalysis 2017, 29, 1887-1893. [CrossRef]

5. Tijunelyte, I.; Betelu, S.; Moreau, J.; Ignatiadis, I.; Berho, C.; Lidgi-Guigui, N.; Guenin, E.; David, C.; Vergnole, S.; Rinnert, E.; et al. Diazonium salt-based surface-enhanced Raman spectroscopy nanosensor: Detection and quantitation of aromatic hydrocarbons in water samples. Sensors 2017, 17, 1198. [CrossRef] [PubMed]

6. Adebiyi, F.M.; Oluyemi, E.A.; Adeyemi, A.F.; Akande, A.A.; Ajayi, O.S. A measurement of selected polycyclic aromatic hydrocarbons in petroleum product contaminated soils using a gas chromatograph. Pet. Sci. Technol. 2014, 33, 62-71. [CrossRef]

7. Bansal, V.; Kumar, P.; Kwon, E.E.; Kim, K.-H. Review of the quantification techniques for polycyclic aromatic hydrocarbons (PAHs) in food products. Crit. Rev. Food Sci. Nutr. 2017, 57, 3297-3312. [CrossRef] [PubMed] 
8. Pena, E.A.; Ridley, L.M.; Murphy, W.R.; Sowa, J.R.; Bentivegna, C.S. Detection of polycyclic aromatic hydrocarbons (PAHs) in raw menhaden fish oil using fluorescence spectroscopy: Method development. Environ. Toxicol. Chem. 2015, 34, 1946-1958. [CrossRef] [PubMed]

9. Wang, Y.; Meng, L.; Pittman, E.N.; Etheredge, A.; Hubbard, K.; Trinidad, D.A.; Kato, K.; Ye, X.; Calafat, A.M. Quantification of urinary mono-hydroxylated metabolites of polycyclic aromatic hydrocarbons by on-line solid phase extraction-high performance liquid chromatography-tandem mass spectrometry. Anal. Bioanal. Chem. 2017, 409, 931-937. [CrossRef] [PubMed]

10. Guo, L.; Tan, S.; Li, X.; Lee, H.K. Fast automated dual-syringe based dispersive liquid-liquid microextraction coupled with gas chromatography-mass spectrometry for the determination of polycyclic aromatic hydrocarbons in environmental water samples. J. Chromatogr. A 2016, 1438, 1-9. [CrossRef] [PubMed]

11. Saini, S.S.; Kabir, A.; Rao, A.L.J.; Malik, A.K.; Furton, K.G. A novel protocol to monitor trace levels of selected polycyclic aromatic hydrocarbons in environmental water using fabric phase sorptive extraction followed by high performance liquid chromatography-fluorescence detection. Separations 2017, 4, 22. [CrossRef]

12. Mollahosseini, A.; Rokue, M.; Mojtahedi, M.M.; Toghroli, M.; Kamankesh, M.; Motaharian, A. Mechanical stir bar sorptive extraction followed by gas chromatography as a new method for determining polycyclic aromatic hydrocarbons in water samples. Microchem. J. 2016, 126, 431-437. [CrossRef]

13. Benede, J.L.; Anderson, J.L.; Chisvert, A. Trace determination of volatile polycyclic aromatic hydrocarbons in natural waters by magnetic ionic liquid-based stir bar dispersive liquid microextraction. Talanta 2018, 176, 253-261. [CrossRef] [PubMed]

14. Dickert, F.L.; Tortschanoff, M.; Bulst, W.E.; Fischerauer, G. Molecularly imprinted sensor layers for the detection of polycyclic aromatic hydrocarbons in water. Anal. Chem. 1999, 71, 4559-4563. [CrossRef]

15. Berho, C.; Claude, B.; Coisy, E.; Togola, A.; Bayoudh, S.; Morin, P.; Amalric, L. Laboratory calibration of a POCIS-like sampler based on molecularly imprinted polymers for glyphosate and AMPA sampling in water. Anal. Bioanal. Chem. 2017, 409, 2029-2035. [CrossRef] [PubMed]

16. Lu, X.; Yang, Y.; Zeng, Y.; Li, L.; Wu, X. Rapid and reliable determination of p-nitroaniline in wastewater by molecularly imprinted fluorescent polymeric ionic liquid microspheres. Biosens. Bioelectron. 2018, 99, 47-55. [CrossRef] [PubMed]

17. Foguel, M.V.; Ton, X.-A.; Zanoni, M.V.B.; Sotomayor, M.D.P.T.; Haupt, K.; Tse Sum Bui, B. A molecularly imprinted polymer-based evanescent wave fiber optic sensor for the detection of basic red 9 dye. Sens. Actuators B Chem. 2015, 218, 222-228. [CrossRef]

18. Zhou, Z.; Li, T.; Xu, W.; Huang, W.; Wang, N.; Yang, W. Synthesis and characterization of fluorescence molecularly imprinted polymers as sensor for highly sensitive detection of dibutyl phthalate from tap water samples. Sens. Actuators B Chem. 2017, 240, 1114-1122. [CrossRef]

19. Hao, T.; Wei, X.; Nie, Y.; Xu, Y.; Yan, Y.; Zhou, Z. An eco-friendly molecularly imprinted fluorescence composite material based on carbon dots for fluorescent detection of 4-nitrophenol. Microchim. Acta 2016, 183, 2197-2203. [CrossRef]

20. Wu, Y.-T.; Liu, Y.-J.; Gao, X.; Gao, K.-C.; Xia, H.; Luo, M.-F.; Wang, X.-J.; Ye, L.; Shi, Y.; Lu, B. Monitoring bisphenol $\mathrm{A}$ and its biodegradation in water using a fluorescent molecularly imprinted chemosensor. Chemosphere 2015, 119, 515-523. [CrossRef] [PubMed]

21. Yaqub, S.; Latif, U.; Dickert, F.L. Plastic antibodies as chemical sensor material for atrazine detection. Sens. Actuators B Chem. 2011, 160, 227-233. [CrossRef]

22. Anirudhan, T.S.; Alexander, S. Multiwalled carbon nanotube based molecular imprinted polymer for trace determination of 2,4-dichlorophenoxyaceticacid in natural water samples using a potentiometric method. Appl. Surf. Sci. 2014, 303, 180-186. [CrossRef]

23. Algieri, C.; Drioli, E.; Guzzo, L.; Donato, L. Bio-mimetic sensors based on molecularly imprinted membranes. Sensors 2014, 14, 13863-13912. [CrossRef] [PubMed]

24. Latif, U.; Dickert, F.L. Conductometric sensors for monitoring degradation of automotive engine oil. Sensors 2011, 11, 8611-8625. [CrossRef] [PubMed]

25. Organization, W.H. Guidelines for Drinking-Water Quality; World Health Organization: Geneva, Switzerland, 2004; Volume 1.

26. Marr, I.; Reiß, S.; Hagen, G.; Moos, R. Planar Zeolite Film-Based Potentiometric Gas Sensors Manufactured by a Combined Thick-Film and Electroplating Technique. Sensors 2011, 11, 7736-7748. [CrossRef] [PubMed] 
27. Xu, M.; Wang, R.; Li, Y. Rapid detection of Escherichia coli O157:H7 and Salmonella Typhimurium in foods using an electrochemical immunosensor based on screen-printed interdigitated microelectrode and immunomagnetic separation. Talanta 2016, 148, 200-208. [CrossRef] [PubMed]

28. Lin, C.W.; Hwang, B.J.; Lee, C.R. Methanol sensors based on the conductive polymer composites from polypyrrole and poly (vinyl alcohol). Mater. Chem. Phys. 1998, 55, 139-144. [CrossRef]

29. Mujahid, A.; Aigner, S.; Dickert, F.L. Micro-structured interdigital capacitors with synthetic antibody receptors for ABO blood-group typing. Sens. Actuators B Chem. 2017, 242, 378-383. [CrossRef] 